\title{
An easy entry to novel early-late oligonuclear transition metal complexes containing $\pi$-conjugated systems
}

\author{
Stephan Back ${ }^{\mathrm{a}}$, Wolfgang Frosch ${ }^{\mathrm{a}}$, Ignacio del Río ${ }^{\mathrm{b}}$, Gerard van Koten ${ }^{\mathrm{b}}$, Heinrich Lang ${ }^{\mathrm{a}, *}$ \\ ${ }^{a}$ Technische Universität Chemnitz, Institut für Chemie, Lehrstuhl Anorganische Chemie, Straße der Nationen 62, 09111 Chemnitz, Germany \\ ${ }^{\mathrm{b}}$ Debye Institute, Department of Metal-Mediated Synthesis, Utrecht University, Padualaan 8, 3584 CH Utrecht, The Netherlands
}

Received 24 September 1999

\begin{abstract}
Heterotrinuclear $\mathrm{Ti}-\mathrm{Cu}-\mathrm{Ru}$ (5) and heterotetranuclear $\mathrm{Ti}-\mathrm{Cu}-\mathrm{Pt}-\mathrm{Fe}$ (7) containing complexes are accessible by using $\left\{[\mathrm{Ti}]\left(\mathrm{C} \equiv \mathrm{C}^{t} \mathrm{Bu}\right)_{2}\right\} \mathrm{CuMe}(\mathbf{1})\left([\mathrm{Ti}]=\left(\eta^{5}-\mathrm{C}_{5} \mathrm{H}_{4} \mathrm{SiMe}_{3}\right)_{2} \mathrm{Ti}\right)$ as key molecule; in $\mathbf{5}$ and $\mathbf{7}$, the corresponding early and late transition metal atoms are linked by $\pi$-conjugated organic moieties.

(C) 1999 Elsevier Science S.A. All rights reserved.

Keywords: Heterometallic complexes; Conjugated ligand complexes; $\pi$-Tweezer complexes; Early-late complexes; Transition metal complexes; Oligonuclear complexes
\end{abstract}

\section{Introduction}

Since the early work of Creutz and Taube (see Ref. [1]), there has been a growing interest in the synthesis, chemical and physical properties of transition metal (TM) complexes in which a $\pi$-conjugated organic ligand spans the corresponding TM atoms [2-6]. Besides homometallic complexes [710], a number of heterometallic species, which mainly feature late TM ions, are also known [11-16]. In contrast, little is known about early-late TM complexes [17-19]. We report in this communication, the preparation of TM complexes of the latter type containing up to four different TM centres.

\section{Results and discussion}

A key step in the synthesis of heteronuclear TM complexes in which the corresponding early and/or late TM centres are spanned by $\pi$-conjugated organic ligands, is the reaction of monomeric alkyne-stabilised $\mathrm{CuMe},\left\{[\mathrm{Ti}]\left(\mathrm{C} \equiv \mathrm{C}^{\mathrm{t}} \mathrm{Bu}\right)_{2}\right\}$ CuMe (1) [20] $\left([\mathrm{Ti}]=\left(\eta^{5}-\mathrm{C}_{5} \mathrm{H}_{4} \mathrm{SiMe}_{3}\right)_{2} \mathrm{Ti}\right)$, with $\mathrm{CH}-$ acidic organic molecules. Thus, complex 1 reacted with an equimolar amount of $\mathrm{HC} \equiv \mathrm{CC}_{6} \mathrm{H}_{4} \mathrm{CN}-4$ (2) [21] at $-70^{\circ} \mathrm{C}$ to yield the yellow $\mathrm{Ti}-\mathrm{Cu}$ acetylide complex $\left\{[\mathrm{Ti}]\left(\mathrm{C} \equiv \mathrm{C}^{\mathrm{t} B u}\right)_{2}\right\} \mathrm{CuC} \equiv \mathrm{CC}_{6} \mathrm{H}_{4} \mathrm{CN}-4$ (3) (Scheme 1).

\footnotetext{
* Corresponding author. Tel.: + 49371531 1200; fax: + 49371531 1833;
} e-mail: heinrich.lang@ chemie.tu-chemnitz.de
The heterodinuclear complex 3 contains a $\mathrm{CN}$ group which can further coordinate. When $\mathbf{3}$ is treated with

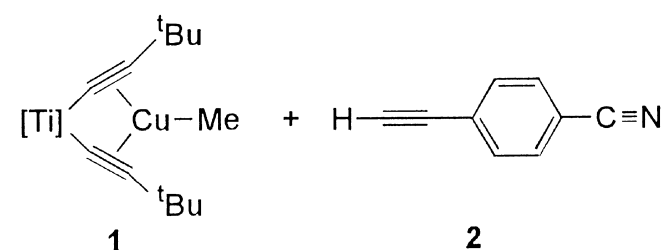

1
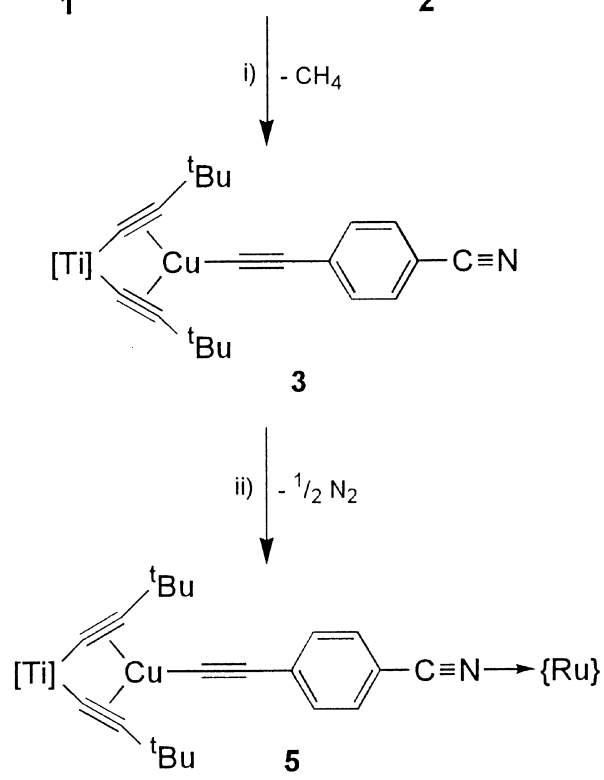

Scheme 1. Preparation of complexes 3 and 5. (i) $0^{\circ} \mathrm{C}$, n-pentane; (ii) $1 / 2$ $\{\mathrm{Ru}\} \mathrm{N} \equiv \mathrm{N}\{\mathrm{Ru}\}, 0^{\circ} \mathrm{C}, \mathrm{THF}$. 
$\{\mathrm{Ru}\} \mathrm{N} \equiv \mathrm{N}\{\mathrm{Ru}\} \quad(4) \quad\left(\{\mathrm{Ru}\}=\left[\eta^{3}-\operatorname{mer}-\left(2,6-\left\{\mathrm{Me}_{2} \mathrm{NCH}_{2}\right\}_{2^{-}}\right.\right.\right.$ $\left.\mathrm{C}_{5} \mathrm{H}_{3} \mathrm{~N}\right\} \mathrm{RuCl}_{2}$ ] ) [22] in molar ratio of $2: 1$ in $\mathrm{THF}$ at $0^{\circ} \mathrm{C}$, $\mathrm{N}_{2}$ is eliminated and heterotrinuclear $\mathbf{5}$ is formed (Scheme 1) 1 .

An access to a heterotetranuclear complex is given by joining dinuclear 1 with the preformed late-late $\mathrm{Pt}-\mathrm{Fe}$ organometallic acetylide $\mathrm{HC} \equiv \mathrm{C}\{\mathrm{Pt}\} \mathrm{C} \equiv \mathrm{CFc} \quad(\mathbf{6}) \quad(\mathrm{HC} \equiv \mathrm{C}\{\mathrm{Pt}\}=$ $\left[\mathrm{Pt}\left(\mathrm{C}_{6} \mathrm{H}_{2}\left\{\mathrm{CH}_{2} \mathrm{NMe}_{2}\right\}_{2}-2,6-\{\mathrm{C} \equiv \mathrm{CH}\}-4\right], \quad \mathrm{Fc}=\left(\eta^{5}-\mathrm{C}_{5} \mathrm{H}_{4}\right)-\right.$ $\left.\mathrm{Fe}\left(\eta^{5}-\mathrm{C}_{5} \mathrm{H}_{5}\right)\right)$ [23]. In THF at $0^{\circ} \mathrm{C}$, the Ti-Cu-Pt-Fe complex $\left\{[\mathrm{Ti}]\left(\mathrm{C} \equiv \mathrm{C}^{\mathrm{t}} \mathrm{Bu}\right)_{2}\right\} \mathrm{CuC} \equiv \mathrm{C}\{\mathrm{Pt}\} \mathrm{C} \equiv \mathrm{CFc}(7)$ is obtained in almost quantitative yield accompanied by the loss of $\mathrm{CH}_{4}$ (Eq. (1) $)^{2}$.

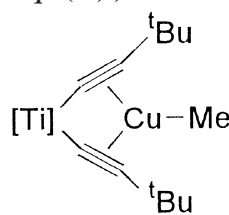

1

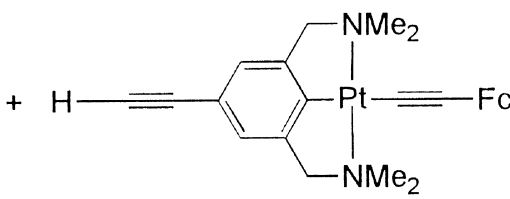

6<smiles>[Y]C(C)C</smiles>

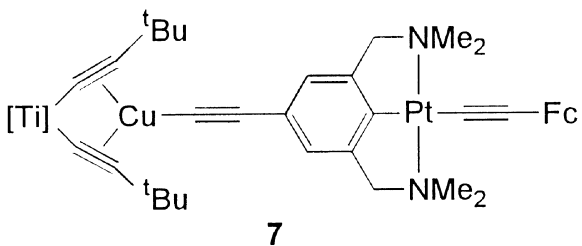

The heterometallic complexes $\mathbf{5}$ and $\mathbf{7}$ are less stable as compared to $\mathbf{1}$ [20], 3 or $\mathbf{6}$ [23].

The formation of $\mathbf{5}$ and $\mathbf{7}$ has been inferred through the IR spectroscopic data. The $\mathrm{C} \equiv \mathrm{N}$ stretching vibration for $\mathbf{5}$ has been shifted to lower wavenumbers $\left(2208 \mathrm{~cm}^{-1}\right)$ as compared to that of $\mathbf{3}\left(2220 \mathrm{~cm}^{-1}\right)$, indicating the $\mathrm{N}$-coordination

\footnotetext{
${ }^{1}$ Synthesis of $5.33 \mathrm{mg}(0.05 \mathrm{mmol})$ of 3 [26] and $17 \mathrm{mg}(0.02 \mathrm{mmol})$ of 4 [21] were dissolved in $10 \mathrm{ml} \mathrm{THF}\left(0^{\circ} \mathrm{C}\right)$. After stirring for $4 \mathrm{~h}$ at $0^{\circ} \mathrm{C}$ the mixture was evaporated to dryness and washed with $\mathrm{n}$-pentane $(5 \times 5$ $\mathrm{ml}$ ) and then dried in vacuo to yield 5 (40 mg, $0.04 \mathrm{mmol}, 80 \%)$; m.p. $112^{\circ} \mathrm{C}$ (decomp.). IR ( $\left.\mathrm{KBr}\right)\left(\mathrm{cm}^{-1}\right): \nu 2208(\mathrm{CN}), 2080,1922(\mathrm{C} \equiv \mathrm{C})$. ${ }^{1} \mathrm{H} \mathrm{NMR}\left(250.13 \mathrm{MHz}, \mathrm{CDCl}_{3}\right): \delta 0.22\left(\mathrm{~s}, 18 \mathrm{H}, \mathrm{SiMe}_{3}\right), 1.38(\mathrm{~s}, 18 \mathrm{H}$, $\left.{ }^{\mathrm{t}} \mathrm{Bu}\right), 2.52\left(\mathrm{~s}, 12 \mathrm{H}, \mathrm{NMe}_{2}\right), 4.01\left(\mathrm{~s}, 4 \mathrm{H}, \mathrm{CH}_{2}\right), 5.95\left(\mathrm{t},{ }^{3} J_{\mathrm{HH}}=1.3 \mathrm{~Hz}\right.$, $\left.\mathrm{C}_{5} \mathrm{H}_{4}\right), 6.01\left(\mathrm{t},{ }^{3} \mathrm{~J}_{\mathrm{HH}}=1.3 \mathrm{~Hz}, \mathrm{C}_{5} \mathrm{H}_{4}\right), 7.1-7.8\left(\mathrm{~m}, 3 \mathrm{H}, \mathrm{C}_{5} \mathrm{H}_{3} \mathrm{~N}\right)$.

${ }^{2}$ Synthesis of 7 . To 1 ( $\left.100 \mathrm{mg}, 0.18 \mathrm{mmol}\right)$ [20] in THF $\left(50 \mathrm{ml}, 25^{\circ} \mathrm{C}\right)$, 6 [23] (110 mg, $0.18 \mathrm{mmol}$ ) was added in one portion. After stirring for $1 \mathrm{~h}$, the mixture was filtered through a pad of Celite. Then, all volatiles were removed in vacuo to yield 7 (200 mg, $0.17 \mathrm{mmol}, 95 \%)$; m.p. $176^{\circ} \mathrm{C}$ (decomp.). IR ( KBr) $\left(\mathrm{cm}^{-1}\right)$ : 2080, 2071, 1942 (C $\left.\equiv \mathrm{C}\right) .{ }^{1} \mathrm{H}$ NMR (250.13 $\left.\mathrm{MHz}, \mathrm{CDCl}_{3}\right): \delta 0.27\left(\mathrm{~s}, 18 \mathrm{H}, \mathrm{SiMe}_{3}\right), 1.38\left(\mathrm{~s}, 18 \mathrm{H},{ }^{\mathrm{t}} \mathrm{Bu}\right), 3.20(\mathrm{~s}$, $\left.{ }^{3} J_{\mathrm{PtH}}=46.2 \mathrm{~Hz}, 12 \mathrm{H}, \mathrm{NMe}_{2}\right), 4.05\left(\mathrm{t},{ }^{3} J_{\mathrm{HH}}=1.2 \mathrm{~Hz}, 2 \mathrm{H}, \mathrm{C}_{5} \mathrm{H}_{4}\right), 4.08(\mathrm{~s}$, $\left.4 \mathrm{H}, \mathrm{CH}_{2}\right), 4.16\left(\mathrm{~s}, 5 \mathrm{H}, \mathrm{C}_{5} \mathrm{H}_{5}\right), 4.37\left(\mathrm{t},{ }^{3} J_{\mathrm{HH}}=1.2 \mathrm{~Hz}, 2 \mathrm{H}, \mathrm{C}_{5} \mathrm{H}_{4}\right), 5.49(\mathrm{t}$, $\left.{ }^{3} J_{\mathrm{HH}}=1.5 \mathrm{~Hz}, 2 \mathrm{H}\right), 5.69\left(\mathrm{t},{ }^{3} J_{\mathrm{HH}}=1.5 \mathrm{~Hz}, 2 \mathrm{H}\right), 7.03\left(\mathrm{~s}, 2 \mathrm{H}, \mathrm{C}_{6} \mathrm{H}_{2}\right)$. ${ }^{13} \mathrm{C}\left\{{ }^{1} \mathrm{H}\right\}$ NMR $\left(62.895 \mathrm{MHz}, \mathrm{CDCl}_{3}\right): \delta 0.3\left(\mathrm{SiMe}_{3}\right), 31.1\left({ }^{\mathrm{t}} \mathrm{Bu}\right), 32.0(\mathrm{C} /$ ${ }^{\mathrm{t}} \mathrm{Bu}$ ), $55.3\left(\mathrm{NMe}_{2}\right), 67.1\left(\mathrm{C}_{5} \mathrm{H}_{4}\right), 68.9\left(\mathrm{C}_{5} \mathrm{H}_{5}\right), 70.5\left(\mathrm{C}_{5} \mathrm{H}_{4}\right), 75.2$ (ipso$\left.\mathrm{C}, \mathrm{C}_{5} \mathrm{H}_{4}\right), 79.6\left(\mathrm{CH}_{2}\right), 110.2\left(\mathrm{C}_{5} \mathrm{H}_{4}\right), 113.5\left(\mathrm{C}_{5} \mathrm{H}_{4}\right), 113.9(\mathrm{CuC} \equiv \mathrm{C})$, 115.2 (ipso-C, $\left.\mathrm{C}_{5} \mathrm{H}_{4}\right), 116.1(\mathrm{CuC} \equiv \mathrm{C}), 123.4(\mathrm{C} \equiv \mathrm{CSi}), 138.2\left(\mathrm{C}_{6} \mathrm{H}_{2}\right)$, 144.2 (ipso-C, $\mathrm{C}_{6} \mathrm{H}_{2}$ ), 146.1 ( $\mathrm{TiC}$ ), 168.1 (ipso-C, $\mathrm{C}_{6} \mathrm{H}_{2}$ ); due to the instability of $\mathbf{7}$ in solution the carbon signals of $\mathrm{PtC} \equiv \mathrm{C}$ could not be detected.
}

of the $\mathrm{CN}$ group to ruthenium. A similar observation has been made for $\{\mathrm{Ru}\} \mathrm{N} \equiv \mathrm{CPh}[24]$ showing a slight decrease in bond order in $\mathrm{C} \equiv \mathrm{N}$. Besides the $\nu_{(\mathrm{C} \equiv \mathrm{N})}$ frequency, two typical $\mathrm{C} \equiv \mathrm{C}$ stretching vibrations have been found at $1922 \mathrm{~cm}^{-1}$ ( $\left.\mathrm{TiC} \equiv \mathrm{C}^{\mathrm{t}} \mathrm{Bu}\right)$ and $2080 \mathrm{~cm}^{-1}\left(\mathrm{CuC} \equiv \mathrm{CC}_{6} \mathrm{H}_{4}\right)$. These two absorptions are in characteristic regions observed for tweezer-stabilised $\mathrm{Cu}(\mathrm{I})$-acetylides [25,26]. Complex 7 exhibits $\nu_{(\mathrm{C} \equiv \mathrm{C})}$ frequencies in this region for the corresponding organometallic acetylide units $\mathrm{TiC} \equiv \mathrm{C}^{\mathrm{t}} \mathrm{Bu}\left(1942 \mathrm{~cm}^{-1}\right)$, $\mathrm{PtC} \equiv \mathrm{CFc}\left(2071 \mathrm{~cm}^{-1}\right)$ and $\mathrm{CuC} \equiv \mathrm{CC}_{6} \mathrm{H}_{2}\left(2080 \mathrm{~cm}^{-1}\right)$.

With the exception of the acetylide linkage, in the ${ }^{13} \mathrm{C}\left\{{ }^{1} \mathrm{H}\right\}$ NMR of 7, the signals of the respective organic fragments are found at chemical shifts typical for the independent molecules $\mathbf{1}$ and $\mathbf{6}$. However, adequate ${ }^{13} \mathrm{C}\left\{{ }^{1} \mathrm{H}\right\}$ NMR studies for $\mathbf{5}$ could not be carried out due to its instability in solution. This decomposition leads to a mixture of products of which $\left\{[\mathrm{Ti}]\left(\mathrm{C} \equiv \mathrm{C}^{\mathrm{t}} \mathrm{Bu}\right)_{2}\right\} \mathrm{CuCl}[25]$ could be separated and characterised unequivocally by spectroscopic means.

In contrast thereto, ${ }^{1} \mathrm{H}$ NMR studies of 5 reveal that the ortho- $\mathrm{CH}_{2} \mathrm{NMe}_{2}$ substituents of the $\mathrm{C}_{5} \mathrm{H}_{3} \mathrm{~N}\left(\mathrm{CH}_{2} \mathrm{NMe}_{2}\right)_{2}-2,6$ ligand remain datively bonded to the $\mathrm{Ru}$ (II) centre. The respective resonance signals are shifted to lower field by $0.5 \mathrm{ppm}\left(\mathrm{CH}_{2}\right)$ and $0.3 \mathrm{ppm}\left(\mathrm{NMe}_{2}\right)$ upon $\eta^{1}-N$-coordination of the $\mathrm{C} \equiv \mathrm{N}$ moiety [24].

The result of this study shows that organic and organometallic $\pi$-systems, such as $\mathrm{TiC} \equiv \mathrm{C}^{\mathrm{t}} \mathrm{Bu}, \mathrm{C}_{6} \mathrm{H}_{2}\left(\mathrm{CH}_{2} \mathrm{NMe}_{2}\right)_{2}-$ 2,6- $(\mathrm{C} \equiv \mathrm{C})-4, \mathrm{C} \equiv \mathrm{CC}_{6} \mathrm{H}_{4} \mathrm{CN}-4$ and $\mathrm{C} \equiv \mathrm{CC}_{5} \mathrm{H}_{4}$, can act as linkage for different early and/or late TM complexes. This gives an entry into the synthesis of novel heterometallic complexes with the TM centres in different coordination modes.

\section{Acknowledgements}

This work was supported by the Volkswagen-Stiftung, Fonds der Chemischen Industrie and Konrad-Adenauer-Stiftung (W.F.).

\section{References}

[1] C. Creutz, Prog. Inorg. Chem. 30 (1983) 1.

[2] D.R. Kanis, P.G. Lacroix, M.A. Ratner, T.J. Marks, J. Am. Chem. Soc. 116 (1994) 10089.

[3] M.B. Sponsler, Organometallics 14 (1995) 1920.

[4] D. Astruc, Acc. Chem. Res. 30 (1997) 383.

[5] P. Belanzoni, N. Re, A. Sgamellotti, C. Floriani, J. Chem. Soc., Dalton Trans. (1998) 1825.

[6] N. Re, A. Sgamellotti, C. Floriani, J. Chem. Soc., Dalton Trans. (1998) 2521.

[7] F. Coat, C. Lapinte, Organometallics 15 (1996) 477.

[8] K. Heinze, G. Huttner, O. Walter, Eur. J. Inorg. Chem. (1999) 513.

[9] W.Y. Wong, W.K. Wong, P.R. Raithby, J. Chem. Soc., Dalton Trans. (1998) 2761.

[10] A. Buttinelli, E. Viola, E. Antonelli, C. Lo Sterzo, Organometallics 17 (1998) 2574

[11] S. Sakanishi, D.A. Bardwell, S. Couchman, J.C. Jeffery, J.A. McCleverty, M.D. Ward, J. Organomet. Chem. 528 (1997) 35.

[12] J. Mata, S. Uriel, E. Peris, R. Llusar, S. Houbrechts, A. Persoons, J. Organomet. Chem. 562 (1998) 197. 
[13] S.B. Falloon, S. Szafert, A.M. Arif, J.A. Gladysz, Chem. Eur. J. 4 (1998) 1033.

[14] U. Siemeling, U. Vorfeld, B. Neumann, H.G. Stammler, P. Zanello, F. Fabrizi de Biani, Eur. J. Inorg. Chem. (1999) 1.

[15] S. Le Stang, D. Lenz, F. Paul, C. Lapinte, J. Organomet. Chem. 572 (1999) 189.

[16] T.Y. Liu, Y.J. Chen, C.C. Tai, K.S. Kwan, Inorg. Chem. 38 (1999) 674.

[17] F.R. Lembke, D.J. Szallda, R.M. Bullock, J. Am. Chem. Soc. 113 (1991) 8466.

[18] Y. Hayashi, M. Osawa, K. Kobayashi, Y. Wakatsuki, Chem. Commun. (1996) 1617.

[19] S. Back, R.A. Gossage, I. del Río, G. Rheinwald, H. Lang, G. van Koten, J. Organomet. Chem. 582 (1999) 126 and Refs. therein.
[20] H. Lang, K. Köhler, L. Zsolnai, M. Büchner, A. Driess, G. Huttner, J. Strähle, Organometallics 18 (1999) 598.

[21] S. Takahashi, Y. Kuroyama, K. Sonogashira, N. Hagihara, Synthesis (1980) 627.

[22] R.A.T.M. Abbenhuis, I. del Río, M.M. Berghoef, J. Boersma, N. Veldman, A.L. Spek, G. van Koten, Inorg. Chem. 37 (1998) 1749.

[23] S. Back, R.A. Gossage, H. Lang, G. van Koten, Eur. J. Inorg. Chem. (1999) submitted for publication.

[24] I. del Río, S. Back, M.S. Hannu, G. Rheinwald, H. Lang, G. van Koten, Inorg. Chim. Acta (1999) submitted for publication.

[25] H. Lang, G. Rheinwald, J. Prakt. Chem. 341 (1999) 1 and Refs. therein.

[26] W. Frosch, S. Back, G. Rheinwald, H. Lang, J. Organomet. Chem. (1999) submitted for publication. 